\title{
RING EXTENSIONS AND ESSENTIAL MONOMORPHISMS
}

\author{
TILMANN WÜRFEL
}

\begin{abstract}
We study pairs of rings $R \subset S$ such that $\operatorname{Hom}_{R}(S,-): R$-Mod $\rightarrow S$-Mod preserves essential monomorphisms. We obtain a complete characterization of such a pair in case $S$ is a torsion-free algebra over a Noetherian domain $R \neq Q \operatorname{Quot}(R) ; S$ is then a left ideally finite $R$-algebra. The rings $R$ such that every ring extension $R \subset S$ satisfies the above condition are subdirect sums of certain Artinian rings. Furthermore, we study a generalization of trivial ring extensions and show that the center of a semi-Artinian ring is again semi-Artinian.
\end{abstract}

Let $R \subset S$ be rings with $1_{S} \in R$. This paper is concerned with the question when the functor $\operatorname{Hom}_{R}(S,-): R$-Mod $\rightarrow S$-Mod preserves essential monomorphisms or, equivalently, injective envelopes (condition (E)). D. Eisenbud has shown [1] that this is the case if $S$ is finitely generated as an $R$-module by elements which centralize $R$, and an argument by Formanek and Jategaonkar [2] shows that the condition $R s=s R$ for the generating elements $s$ is sufficient; furthermore, the condition that ${ }_{R} S$ be finitely generated can be weakened (Example 2). Another type of example is furnished by the construction $S=R \times M$, where $M$ is an $R$-bimodule and multiplication is defined by an "associative" mapping $M \otimes_{R} M \rightarrow R$; Example 7 generalizes this situation. Our main result is Theorem 3: If $R$ is a Noetherian domain but not a field and $S$ a torsion-free $R$-algebra, then $R \subset S$ satisfies condition (E) if and only if $S$ is an ideally finite $R$-algebra, i.e. every nonzero left $S$-module contains a nonzero submodule which is finitely generated over $R$. The proof of the theorem is essentially set-theoretical and the nontrivial part of it is false for non-Noetherian domains $R$. In Proposition 8 we give a short proof of the reflexion of relative injectivity and its consequence for chain conditions for (E)-extensions. We close with two examples involving semi-Artinian rings (Theorems 9, 10).

Finally, let us mention the corresponding problem defined by an $R$-module $M$ and its endomorphism ring $E$. It is easy to see that, if $M$ generates all of its submodules, then $\operatorname{Hom}_{R}(M,-): R$-Mod $\rightarrow E$-Mod preserves essential monomorphisms. Under certain assumptions on $M$ the converse to this fact has been shown in [8, Theorem 2.4]. Our case, however, where we consider rings $R \subset S$ (not $\operatorname{End}\left({ }_{R} S\right)$ ) requires other methods since the multiplicative

Received by the editors April 21, 1977 and, in revised form, July 22, 1977.

AMS (MOS) subject classifications (1970). Primary 16A56, 16A52, 16A46.

Key words and phrases. Ring extensions, essential monomorphisms, injective hulls, descent of injectivity, Noetherian modules, ideally finite algebras, semi-Artinian rings. 
structure of $S$ plays an important role besides its $R$-module structure.

All rings, modules, etc. are assumed to be unitary. For a subgroup $U$ of some $S$-module $M$, denote by $(U: S)$ the largest $S$-submodule of $M$ contained in $U$.

Proposition 1. For any pair of rings $R \subset S$ the following conditions are equivalent.

(E) The functor $\operatorname{Hom}_{R}(S,-): R$-Mod $\rightarrow S$-Mod preserves essential monomorphisms.

(E') For every essential $R$-submodule $U$ of a nonzero left $S$-module $M,(U$ : $S$ ) is not zero (resp. essential in ${ }_{S} M$ ).

Proof. (E) $\Rightarrow\left(\mathrm{E}^{\prime}\right)$. Consider $M$ as an $S$-submodule of $\operatorname{Hom}_{R}(S, M)$. Then $(U: S)=M \cap \operatorname{Hom}_{R}(S, U)$ is essential in $M .\left(\mathrm{E}^{\prime}\right) \Rightarrow(\mathrm{E})$. Since $(U: S) \cap N$ $=(U \cap N: S)$ for every $S$-submodule $N$ of $M$, both versions of $\left(\mathrm{E}^{\prime}\right)$ are equivalent. Now let $X$ be essential in ${ }_{R} Y$ and denote by $U$ its inverse image under the mapping $\operatorname{Hom}_{R}(S, Y) \rightarrow Y$ defined by $f \mapsto f(1)$. Then $(U: S)=$ $\operatorname{Hom}_{R}(S, X)$ which proves (E).

Let us call a pair of rings $R \subset S$ an (E)-extension if it satisfies the above conditions. Then, for any intermediate ring $S^{\prime}$ between $R$ and $S, R \subset S^{\prime}$ is also an (E)-extension.

A family $\left(J_{\alpha}\right)_{\alpha<\tau}$ of submodules of some module, indexed by an ordinal $\tau$, is called a continuous chain, if it is increasing and if $J_{\lambda}=\cup_{\alpha<\lambda} J_{\alpha}$ for limit ordinals $\lambda$.

EXAmple 2. Let $R \subset S$ be rings. Assume that ${ }_{s} S_{R}$ contains a continuous chain of submodules $\left(J_{\alpha}\right)_{\alpha<\tau}$ terminating at $J_{\tau}=S$ such that, for all $\alpha<\tau$, $J_{\alpha+1} / J_{\alpha}$ is a sum of $S$-submodules which, as left $R$-modules, are generated by finitely many elements $x_{i}$ satisfying $R x_{i}=x_{i} R$. Then $R \subset S$ is an (E)-extension.

Proof. Let $U$ be an essential $R$-submodule of some ${ }_{s} M \neq(0)$. We may assume that the least ordinal $\alpha$ such that $J_{\alpha} M \neq(0)$ is 1 . Then $J_{1}$ contains a left $S$-ideal $L=R s_{1}+\cdots+R s_{n}$ such that $L M \neq(0)$ and $R s_{i}=s_{i} R$ for all $i$. Say $s_{1} x \neq 0$ for some $x$ in $M$. As in the proof of [2, Theorem 4], by induction on $j \leqslant n$, we can find elements $r_{j} \in R$ such that $(0) \neq\left(R s_{1}\right.$ $\left.+\cdots+R s_{j}\right) r_{j} x \subset U$. Hence $(0) \neq L r_{n} x \subset(U: S)$.

Our main result is the converse to Example 2 in the following special case.

THEOREM 3. Let $R$ be a Noetherian domain different from its quotient field and $A$ an $R$-algebra which is a torsion-free $R$-module. Then the following are equivalent.

(a) $R \subset A$ is an (E)-extension. (b) $A$ contains a continuous chain of ideals $\left(J_{\alpha}\right)_{\alpha \leqslant \tau}$ terminating at $J_{\tau}=A$ such that each quotient $J_{\alpha+1} / J_{\alpha}$ is the sum of its left $A$-submodules which are finitely generated over $R$.

The implication (a) $\Rightarrow$ (b) will follow from the more general Theorem $3^{\prime}$ below. For a domain $R \neq Q \operatorname{Quot}(R)$ let us denote by $i(R)$ the greatest cardinal 
such that every set of less than $i(R)$ nonzero ideals of $R$ has nonzero intersection. Then $\aleph_{0} \leqslant i(R) \leqslant \operatorname{card}(R)$, and $i(R)=\aleph_{0}$ when $R$ is Noetherian. (See [4] for a domain $R$ satisfying $i(R)=\aleph_{1}$.)

TheOREM 3'. Let $R$ be a domain different from its quotient field and a a cardinal $>i(R)$. Further, let $M$ and $N$ be torsion-free $R$-modules and $\left(h_{\alpha}\right)_{\alpha \in \mathfrak{a}} a$ linearly independent family in $\operatorname{Hom}_{R}(M, N)$ such that every essential submodule of $N$ contains the image of some nonzero mapping in $H=\Sigma_{\alpha} R h_{\alpha}$. Then there exists a nonzero $h \in H$ of $\operatorname{rank}(h)<\mathfrak{a}$.

We need two lemmas for the proof. Denote by a an arbitrary infinite cardinal.

LEMMa 4. Let $\left\{f_{\alpha} / \alpha \in \mathfrak{a}\right\} \subset \operatorname{Hom}_{K}(V, W)$ be a linearly independent family of homomorphisms of vector spaces over some field $K$ such that every nonzero $f \in \Sigma_{\alpha} K f_{\alpha}$ has $\operatorname{rank}(f) \geqslant a$. Then there exists a family $\left\{X_{\alpha} / \alpha \in a\right\}$ of subspaces of $V$ satisfying the following conditions.

(a) The sum $\Sigma_{\alpha} f_{\alpha}\left(X_{\alpha}\right)$ is direct. (b) $X_{\alpha} \cap \operatorname{Ker}\left(f_{\alpha}\right)=(0)$ for all $\alpha$. (c) $\operatorname{dim}_{K}\left(\cap X_{\alpha_{i}}\right)=$ a for every finite subset $\left\{\alpha_{i}\right\} \subset a$.

Proof. We need the following fact from linear algebra. If finitely many $g_{1}, \ldots, g_{m} \in \operatorname{Hom}_{K}(U, W)$ are such that the vectors $g_{1}(u), \ldots, g_{m}(u)$ are linearly dependent for every $u \in U$, then there exist $k_{i} \in K$, not all zero, such that $k_{1} g_{1}+\cdots+k_{m} g_{m}$ has finite rank. Let us note the following consequence. If every nonzero linear combination of a linearly independent set $g_{1}, \ldots, g_{m} \in \operatorname{Hom}_{K}(V, W)$ is of rank $\geqslant a$, then every subspace $U$ of $V$ of codimension $<$ a contains a vector $u$ such that $g_{1}(u), \ldots, g_{m}(u)$ are linearly independent.

We are now in a position to construct the spaces $X_{\alpha}$. Denote by $\left\{F_{\alpha} / \alpha \in\right.$ a) the family of all finite nonempty subsets of $a$. By transfinite induction we can define a family $\left\{x_{\alpha} / \alpha \in a\right\} \subset V$ such that each family $\left\{f_{\varepsilon}\left(x_{\alpha}\right) / \varepsilon \in F_{\alpha}\right\}$ is linearly independent and that, denoting by $S_{\alpha}$ its linear span, the sum $\Sigma_{\alpha} S_{\alpha}$ is direct. To see that this definition is possible put $Y=\Sigma_{\alpha<\beta} S_{\alpha}$ for some $\beta \in a$ and set $W=Y \oplus C$ and $U=\cap_{\varepsilon \in F_{\beta}} f_{\varepsilon}^{-1}(C)$. Since $\operatorname{dim}_{K}(Y)<a$, by the preceding remark, we can find $x_{\beta} \in U$ such that $\left\{f_{\varepsilon}\left(x_{\beta}\right) / \varepsilon \in F_{\beta}\right\}$ is linearly independent and the sum $Y+S_{\beta}$ is direct. Finally, let $E_{\varepsilon}=\{\alpha \in$ a $\left./ \varepsilon \in F_{\alpha}\right\}$ and define $X_{\varepsilon}=\Sigma_{\alpha \in E_{\varepsilon}} K x_{\alpha}$ for all $\varepsilon \in$ a. Properties (a), (b), (c) can now be easily checked.

LEMMA 5. Let $R \neq Q$ Quot $(R)$ be a domain and $F$ a torsion-free $R$-module of $\operatorname{rank}(F)=a \geqslant i(R)$. Suppose further a set $\Phi$ of submodules of $F$ to be given, of cardinality $\leqslant \mathfrak{a}$, such that every essential submodule of $F$ contains some $r U$ where $U \in \Phi$ and $r \in R-\{0\}$. Then $\Phi$ must contain a module $U$ of $\operatorname{rank}(U)$ $<a$.

Proof. Let $\Phi=\left\{U_{\alpha} / \alpha \in a\right\}$. By a routine reduction argument, we may restrict ourselves to the case when $F=\bigoplus_{\alpha} R x_{\alpha}$ is free on the basis $\left\{x_{\alpha} / \alpha \in\right.$ 
a $\}$ and $U_{\alpha}=\bigoplus_{\beta} R s_{\alpha \beta} x_{\beta}$ with $s_{\alpha \beta} \in R$. Then let $T_{\alpha}=\left\{\beta \in \mathfrak{a} / s_{\alpha \beta} \neq 0\right\}$ and assume, by way of contradiction, that $\operatorname{rank}\left(U_{\alpha}\right)=\operatorname{card}\left(T_{\alpha}\right)=a$ for all $\alpha \in \mathfrak{a}$. By a set-theoretical argument we can find subsets $S_{\alpha} \subset T_{\alpha}$ such that card $\left(S_{\alpha}\right)$ $=\mathfrak{a}$ and $S_{\alpha} \cap S_{\beta}=\varnothing$ for all $\alpha \neq \beta$. Since $i(R) \leqslant \mathfrak{a}$, there is a subset $\left\{r_{\alpha} / \alpha \in \mathfrak{a}\right\} \subset R-\{0\}$ such that $\cap_{\alpha} R r_{\alpha}=(0)$. Put $r_{\alpha \beta}=r_{j_{\alpha}(\beta)}$ where $j_{\alpha}$ : $S_{\alpha} \rightarrow \mathfrak{a}$ is some bijective mapping and set $E_{\alpha}=\bigoplus_{\beta \in S_{\alpha}} R r_{\alpha \beta} s_{\alpha \beta} x_{\beta}$. Then the direct sum $E=\bigoplus_{\alpha} E_{\alpha}+\bigoplus_{\gamma \in \mathfrak{b}} R x_{\gamma}$ with $\mathfrak{b}=\mathfrak{a}-\cup_{\alpha} S_{\alpha}$ is essential in $F$. Hence there exist $\delta \in a$ and $r \neq 0$ such that $r U_{\delta} \subset E$. Comparing coefficients now yields the contradiction $r \in \cap_{\beta \in S_{\delta}} R r_{\delta \beta}=(0)$.

Proof of Theorem 3'. Suppose that $\operatorname{rank}(h) \geqslant a$ for every nonzero $h \in H$. Then let $K=$ Quot $(R)$ and put $V=K \otimes M, W=K \otimes N$, and $f_{\alpha}=1 \otimes h_{\alpha}$. Let $\left(X_{\alpha}\right)$ be the family of subspaces of $V$ corresponding to the $f_{\alpha}$ 's as described in Lemma 4 and set $Y_{\alpha}=M \cap X_{\alpha}$ and $F_{\alpha}=h_{\alpha}\left(Y_{\alpha}\right)$. By Lemma 4, the submodule $h_{\alpha}\left(Y_{\alpha} \cap \cap{ }_{i} Y_{\alpha_{i}}\right)$ of $F_{\alpha}$ has rank a for every finite subset $\left\{\alpha_{i}\right\}$ of a. Denote by $\Phi_{\alpha}$ the set of all such submodules of $F_{\alpha}$. By Lemma 5, $F_{\alpha}$ must contain an essential submodule $E_{\alpha}$ not containing any of the modules $r U$ with $U \in \Phi_{\alpha}$ and $r \neq 0$. Choose a submodule $C$ of $N$ such that the sum $E=\bigoplus_{\alpha} E_{\alpha}+C$ is direct and essential in $N$ and let $h=r_{1} h_{\alpha_{1}}+\cdots+r_{n} h_{\alpha_{n}}$ with nonzero $r_{i} \in R$ and different $\alpha_{i}$ be such that $h(M) \subset E$. Then $h\left(\cap_{i} Y_{\alpha_{i}}\right)$ $\subset E \cap\left(\bigoplus_{\alpha} F_{\alpha}\right)=\bigoplus_{\alpha} E_{\alpha}$ and, hence, $r_{1} h_{\alpha_{1}}\left(\cap_{i} Y_{\alpha_{1}}\right) \subset E_{\alpha_{1}}$, contradicting the choice of $E_{\alpha_{1}}$.

COROllary 6. Let the domain $R$ satisfy $i(R)=\aleph_{0}$. Let $N$ be a nonzero left module over some $R$-algebra $A$ such that ${ }_{R} N$ is torsion-free and $(U: A)$ is an essential $A$-submodule for every essential $R$-submodule $U$ of $N$. Then $N$ contains a finitely generated $R$-submodule $F$ such that $(F: A) \neq(0)$.

Proof. Consider a nonzero $A$-submodule $X$ of $N$ of least $R$-rank and apply Theorem $3^{\prime}$ to homomorphisms of the form $a \mapsto a x$ for $a \in A$ and $x \in X$.

Proof of Theorem 3. For a left $A$-module $M$, denote by $q(M)$ the sum of its submodules which are finitely generated over $R$. Defining $J_{\alpha+1} / J_{\alpha}=$ $q\left(A / J_{\alpha}\right)$, Corollary 6 shows that $J_{\tau}=A$ for some ordinal $\tau$.

REMARKs. (1) Following [7], let us call an algebra $A$ over an arbitrary commutative ring $R$ left ideally finite if it satisfies condition (b) of Theorem 3 , or, equivalently, if any nonzero left $A$-module contains a nonzero submodule which is finitely generated over $R$. Such an algebra is easily seen to be locally finite.

(2) For a non-Noetherian domain $R$, Theorem 3 , (a) $\Rightarrow($ b), is false, in general, as can be seen by taking the polynomial ring $A=\mathbf{Z}[X]$ and its subring $R=\mathbf{Z}+p A$ for some prime $p$. For an essential $R$-submodule $U$ of some ${ }_{A} M \neq(0)$, we have $(0) \neq N+J U \subset(U: A)$, where $N$ denotes the annihilator of $J=p A$ in $M$. But $A$ is not integral over $R$.

EXAMPLE 7. Let $R \subset S$ be rings and assume that $S_{R}$ contains a submodule $J$ such that $S=R+J$ and, given any sequence $s_{0}, s_{1}, \ldots$, of elements of $J$, there 
is an index $n$ such that $s_{n} s_{n-1} \cdots s_{0} \in R$. Then $R \subset S$ has property (E) and every nonzero left $S$-module $M$ contains a finitely generated $R$-submodule $F$ such that $(F: S) \neq(0)$.

Proof. Suppose $(U: S)=(0)$ for an essential $R$-submodule $U$ of some $s^{M} \neq(0)$. Then $J u \not \subset U$ for every nonzero $u \in U$. Fixing such an element $u$ and using the fact that $U$ is $R$-essential we obtain a sequence $s_{0}, s_{1}, \ldots$ from $J$ such that $s_{n} s_{n-1} \cdots s_{0} u \notin U$ for all $n$, a contradiction. To prove the second statement assume $(F: S)=(0)$ for every f.g. ${ }_{R} F$ in $M$ and let $x \in M-\{0\}$. In a way similar to the preceding argument we can find elements $s_{n} \in J$ and f.g. $R$-submodules $F_{n}$ such that $x \in F_{n}$ and $s_{n} s_{n-1} \cdots s_{0} x \notin F_{n}$ for all $n$, which gives the contradiction.

REMARK. Let $R \subset S$ be as above and assume that, in addition, $J$ is an ideal in $S$. Then, as for trivial ring extensions, every injective left $S$-module can be shown to be isomorphic to $\operatorname{Hom}_{R}(S, X)$ for some ${ }_{R} X$.

The results of [1] and [2] on descent of chain condition for ring extensions of the type of Example 2 apply also to those described in Example 7. Let us mention the following footnote to Eisenbud's paper.

Proposition 8. Let $R \subset S$ be rings. (a) If condition (E) holds and if ${ }_{S} M$ and ${ }_{R} X$ are such that $\operatorname{Hom}_{R}(S, X)$ is $M$-injective, then $X$ is ${ }_{R} M$-injective (condition (I)). (b) Assume that condition (I) holds and that every nonzero ${ }_{s} M$ contains some f.g. $R$-submodule $F$ such that $(F: S) \neq(0)$. Then any Noetherian left $S$-module is Noetherian as an $R$-module.

Proof. (a) Recall a module $X$ being $Y$-injective if every homomorphism $Z \rightarrow X, Z$ a submodule of $Y$, can be extended to $Y$. Since this is the case if and only if $\operatorname{Hom}(Y, X) \rightarrow \operatorname{Hom}(Y, E(X))$ is an isomorphism for $E(X)$ an injective hull of $X$, the statement follows by a straightforward adjointness argument. (b) Let ${ }_{s} M$ be Noetherian. From the second part of the assumption it follows that ${ }_{R} M$ is finitely generated (consider a maximal $(F: S)$ with ${ }_{R} F$ f.g.). By a well-known argument due to Bass it suffices to show ${ }_{R} M$ to be $X$-injective for $X$ an arbitrary direct sum of injective $R$-modules. But $\operatorname{Hom}_{R}(S, X)$ is $M$-injective since every submodule of ${ }_{s} M$ is f.g. over $R$. Thus condition (I) yields the conclusion.

In closing, let us note two examples of (E)-extensions of semi-Artinian rings. The following statement can be expressed by saying that the pair $R \subset S$ in question has property (E).

TheOREM 9. The center $R$ of a left semi-Artinian ring $S$ is semi-Artinian.

Proof. Let $x$ be a central element of an arbitrary ring $S$ with left socle $I$. If $S x^{n} \equiv S x^{n+1}(\bmod I)$ for some $n \geqslant 0$, then $S x^{m}=S x^{m+1}$ for some $m \geqslant 0$. To see this, let $s \in S$ such that $y=x^{n}-s x^{n+1} \in I$. Since $S y$ is Artinian, we get $S y^{k}=S y^{k+1}$ for some $k \geqslant 1$. Centrality of $x$ now yields $x^{n k} \in S x^{n k+1}$, so $m=n k$ does it.

Next we claim that $S x^{n}=S x^{n+1}$ for every $x \in R$ and some $n$ depending 
on $x$. Let $\left(I_{\alpha}\right)_{\alpha<\tau}$ be the Loewy series of ${ }_{S} S$, i.e. the continuous chain of ideals defined by $I_{\tau}=S$ and $I_{\alpha+1} / I_{\alpha}=\operatorname{soc}\left({ }_{s} S / I_{\alpha}\right)$. We must show that the least ordinal $\alpha$ such that $S x^{n} \equiv S x^{n+1}\left(\bmod I_{\alpha}\right)$ for some $n>0$ is zero. If not, $\alpha=\beta+1$. Then consider the ring $S^{\prime}=S / I_{\beta}, x^{\prime}=x+I_{\beta}$, and $I^{\prime}=$ $I_{\alpha} / I_{\beta}$. By the preceding remark it follows that $S^{\prime} x^{\prime m}=S^{\prime} x^{\prime m+1}$ for some $m \geqslant 0$, contradicting the minimality of $\alpha$. It follows that every $x \in R$ satisfies some equation $R x^{n}=R x^{n+1}$ [5, Satz 2.5], i.e. $R$ has Krull dimension zero. Hence every maximal left ideal of $S$ has maximal intersection with $R$. Thus $S$ is a semi-Artinian $R$-module. Q.E.D.

THEOREM 10. For any ring $R$ the following conditions are equivalent.

(a) Every ring extension $R \hookrightarrow S$ has property (E).

(b) $R / t(R), t(R)$ being the torsion part of $(R,+)$, and $R / p R$, for every prime $p$, are semisimple rings.

(c) $R=A \times B$, with $B$ a semisimple $Q$-algebra and $A$ a subring of the product $\Pi_{p} A_{p}$ of Artinian rings $A_{p}$ satisfying $\operatorname{rad}\left(A_{p}\right)=p A_{p}$ for every prime $p$, such that $A$ contains the ideal $I=\bigoplus_{p} A_{p}$ and $A / I$ is semisimple.

Proof. (a) $\Rightarrow$ (b) For any homomorphism of rings $h: R \rightarrow T$ and simple left $T$-module, the induced $R$-module $M_{(h)}$ is semisimple. This can be seen by making $M$ a simple module over $S=R \times T$ and applying Proposition 8(a) to $R \hookrightarrow S$. Thus, any left $R$-module whose ring of endomorphisms contains a subfield must be semisimple. In particular, so are $Q \otimes_{\mathrm{Z}} R / t(R)$ and $R / p R$. $(c) \Rightarrow(b) \Rightarrow(a)$ is easy.

(b) $\Rightarrow$ (c) Denote by $t_{p}(R)$ the $p$-component and by $d(R)$ the divisible part of the additive group of $R$. Since $R / t(R)$ is divisible, the argument from [3, Lemma 2] yields $R=p^{n} R \oplus t_{p}(R)$ for some $n \geqslant 0$. Setting $B=d(R)$ we have $B \cap t(R)=(0)$ since all components of $R$ are bounded. Thus $B$ is a module over $R / t(R)$. These modules are easily seen to be injective over $R$. Thus $R=B \oplus A$ with $A$ a left ideal. $A$ is also a right ideal because the right annihilator of $B$ in $R$ has zero intersection with $B$. Finally, consider the unitary ring $A_{p}=t_{p}(R)$. Since it is bounded as a group and since $A_{p} / p A_{p} \cong$ $R / p R$ is semisimple, it is Artinian with radical $p A_{p}$. The remaining part is obvious.

Remark. Let $A$ be an Artinian ring such that $\operatorname{rad}(A)=p A$ for some prime $p$. Then $A$ is the product of a finite number of full matrix rings over local Artinian rings $A^{\prime}$ satisfying $\operatorname{rad}\left(A^{\prime}\right)=p A^{\prime}$. For more information about these rings $A^{\prime}$, see [6].

\section{REFERENCES}

1. D. Eisenbud, Subrings of Artinian and Noetherian rings, Math. Ann. 185 (1970), 247-249.

2. E. Formanek and A. V. Jategaonkar, Subrings of Noetherian rings, Proc. Amer. Math. Soc. 46 (1974), 181-186.

3. L. Fuchs and K. M. Rangaswami, On generalized regular rings, Math. Z. 107 (1968), 71-81.

4. R. Gilmer, $A$ note on the quotient field of the domain $D[[X]]$, Proc. Amer. Math. Soc. 18 (1967), 1138-1140. 
5. F. Kasch and U. Oberst, Das Zentrum von Ringen mit Kettenbedingungen, Sitzungsber. Bayer. Akad. Wiss. Math.-Natur. Kl. 10 (1970), 161-179.

6. K. R. McLean, p-Rings whase only right ideals are the fully inoariant subgroups, Proc. London Math. Soc. (3) 30 (1975), 445-458.

7. I. Stewart, The Wedderburn-Malceo theorems in a locally finite setting, Arch. Math. 27 (1976), 120-122.

8. B. Zimmermann-Huisgen, Endomorphism rings of self-generators, Pacific J. Math. 61 (1975), 587-602.

Mathematischies Institut der Universttät, Theresienstrasse 39, D-8 München 2, Federal Republic of GrRmany 